Homology, Homotopy and Applications, vol. 22(1), 2020, pp.55-68

\title{
BOX-HOMOTOPY AND THE REDUCTION OF pro*-HTop CATEGORY
}

\author{
NIKOLA KOCEIĆ BILAN AND IVANČICA MIROŠEVIĆ
}

(communicated by Aleksandr Mishchenko)

\begin{abstract}
A new generalized definition of homotopy is proposed, including a new class of continuous mappings that we call box-homotopies. It turns out that to be box-homotopic is an equivalence relation on $\operatorname{Top}(X, Y)$ and $\operatorname{HTop}(X, Y)$, and that it is well adjusted with composition, which allows us to introduce a new category $H_{\square} T o p$, the corresponding quotient category of the category HTop, and consequently, pro- $H_{\square} T o p$. We propose a new functor $\tilde{R}$ from $p r o^{*}-H T o p$ to pro- $H_{\square} T o p$, which represents morphisms in pro*-category as $^{*}$ morphisms in pro-category between more complex objects.
\end{abstract}

\section{Introduction}

In this article we observe a reduced power of a topological space, an object that can be found in studies of Rudin, Kunen and Bankston (see, for example, $[\mathbf{6}, \mathbf{3}]$ or [1]). It is a product of topological space $X$ by itself countably many times, given the box topology, and reduced to a quotient space by an equivalence relation saying that two sequences of elements in $X$ are related if they differ at most on a finite number of coordinates. This quotient space will be denoted by $\tilde{X}$ or $\nabla X$.

We list some of its properties. For instance, it preserves separation properties from the original space. Also, in $\tilde{X}$, intersections of countably many open sets, in other words $G_{\delta}$ sets, are open. In the literature such a space is called a $P$-space in the sense of Gillman-Henriksen.

In the sequel we propose a generalization of the notion of homotopy, a relation that we call box-homotopy and denote by $\sim$. Naturally, all homotopic maps are boxhomotopic, and we provide an example showing that the converse does not hold. Then we construct a new category, $H_{\square} T o p$, with topological spaces as objects and box-homotopy classes of continuous maps as morphisms, as it proves out that boxhomotopy is an equivalence relation on $\operatorname{Top}(X, Y)$, and that it is well adjusted with the composition.

Finally we propose a new functor $\tilde{R}$ from $p^{*}{ }^{*}-H T o p$ to $p r o-H_{\square} T o p$, which repre-

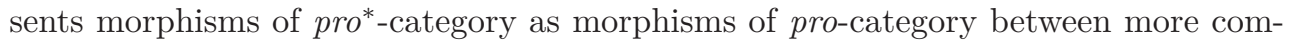
plex objects. For instance, inverse systems in Example 7.2 of [2] are not isomorphic

Received December 3, 2018; published on October 23, 2019.

2010 Mathematics Subject Classification: 55P55, 55Q05, 55N99.

Key words and phrases: reduced product, $P$-space, box-homotopy, pro*-category.

Article available at http://dx.doi.org/10.4310/HHA.2020.v22.n1.a4

Copyright (C) 2019, International Press. Permission to copy for private use granted. 


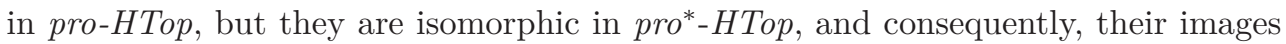
under $\tilde{R}$ are isomorphic in pro- $H_{\square} T o p$.

\section{Preliminaries}

Let us provide a brief survey of the required foreknowledge (see [2]).

Let $\boldsymbol{X}=\left(X_{\lambda}, p_{\lambda \lambda^{\prime}}, \Lambda\right)$ and $\boldsymbol{Y}=\left(Y_{\mu}, q_{\mu \mu^{\prime}}, M\right)$ be two inverse systems in some category $\mathcal{C}$ and $f: M \rightarrow \Lambda$ a function called the index function. A morphism $\left(f, f_{\mu}\right): X \rightarrow$ $\boldsymbol{Y}$ consists of the function $f$ and a set of morphisms $f_{\mu}: X_{f(\mu)} \rightarrow Y_{\mu}$, one for each $\mu \in M$, in $\mathcal{C}$, such that, for every pair $\mu, \mu^{\prime} \in M, \mu \leqslant \mu^{\prime}$, there exists a $\lambda \in \Lambda, \lambda \geqslant$ $f(\mu), f\left(\mu^{\prime}\right)$ such that

$$
f_{\mu} p_{f(\mu) \lambda}=q_{\mu \mu^{\prime}} f_{\mu^{\prime}} p_{f\left(\mu^{\prime}\right) \lambda} .
$$

The category inv-C $\mathcal{C}$ has all inverse systems for objects, and all morphisms $\left(f, f_{\mu}\right)$ between inverse systems as morphisms.

A morphism $\left(f, f_{\mu}\right): \boldsymbol{X} \rightarrow \boldsymbol{Y}$ is equivalent to a morphism $\left(f^{\prime}, f_{\mu}^{\prime}\right): \boldsymbol{X} \rightarrow \boldsymbol{Y}$, denoted by $\left(f, f_{\mu}\right) \sim\left(f^{\prime}, f^{\prime}{ }_{\mu}\right)$, if for every $\mu \in M$ there exists a $\lambda \in \Lambda, \lambda \geqslant f(\mu), f^{\prime}(\mu)$ such that $f_{\mu} p_{f(\mu) \lambda}=f^{\prime}{ }_{\mu} p_{f^{\prime}(\mu) \lambda}$. The category pro-C has all inverse systems for objects, and all equivalence classes $\boldsymbol{f}=\left[\left(f, f_{\mu}\right)\right]$ of all morphisms $\left(f, f_{\mu}\right)$ between inverse systems as morphisms.

There are another two categories with the same objects, but with richer morphism classes, containing the previous two as subcategories and we shall need one of them. Let $\boldsymbol{X}=\left(X_{\lambda}, p_{\lambda \lambda^{\prime}}, \Lambda\right)$ and $\boldsymbol{Y}=\left(Y_{\mu}, q_{\mu \mu^{\prime}}, M\right)$ be two inverse systems in some category $\mathcal{C}$ and $f: M \rightarrow \Lambda$ a function called the index function. A morphism $\left(f, f_{\mu}^{n}\right): \boldsymbol{X} \rightarrow \boldsymbol{Y}$ consists of the function $f$ and a set of morphisms $f_{\mu}^{n}: X_{f(\mu)} \rightarrow Y_{\mu}, n \in \mathbb{N}, \mu \in M$, in $\mathcal{C}$, such that, for every pair $\mu, \mu^{\prime} \in M, \mu \leqslant \mu^{\prime}$, there exists $\lambda \in \Lambda, \lambda \geqslant f(\mu), f\left(\mu^{\prime}\right)$ and $n \in \mathbb{N}$ such that for every $n^{\prime} \geqslant n$,

$$
f_{\mu}^{n^{\prime}} p_{f(\mu) \lambda}=q_{\mu \mu^{\prime}} f_{\mu^{\prime}}^{n^{\prime}} p_{f\left(\mu^{\prime}\right) \lambda} .
$$

The category $i n v^{*}-\mathcal{C}$ has all inverse systems for objects, and all morphisms $\left(f, f_{\mu}^{n}\right)$ between inverse systems as morphisms.

A morphism $\left(f, f_{\mu}^{n}\right): \boldsymbol{X} \rightarrow \boldsymbol{Y}$ is equivalent to a morphism $\left(f^{\prime}, f_{\mu}^{\prime n}\right): \boldsymbol{X} \rightarrow \boldsymbol{Y}$, denoted by $\left(f, f_{\mu}^{n}\right) \sim\left(f^{\prime}, f_{\mu}^{\prime n}\right)$, if for every $\mu \in M$ there exists a $\lambda \in \Lambda, \lambda \geqslant f(\mu), f^{\prime}(\mu)$ and $n \in \mathbb{N}$ such that $f_{\mu}^{n^{\prime}} p_{f(\mu) \lambda}={f^{\prime}}_{\mu}^{n^{\prime}} p_{f^{\prime}(\mu) \lambda}$, for every $n^{\prime} \geqslant n$. The category pro*-C has all inverse systems for objects, and all equivalence classes $\boldsymbol{f}^{*}=\left[\left(f, f_{\mu}^{n}\right)\right]$ of all morphisms $\left(f, f_{\mu}^{n}\right)$ between inverse systems as morphisms.

By a mapping that holds inverse systems fixed, and that sends a morphism $\boldsymbol{f}=$ $\left[\left(f, f_{\mu}\right)\right]$ in pro-C to a morphism $\boldsymbol{f}^{*}=\left[\left(f, f_{\mu}^{n}\right)\right]$ in pro $^{*} \mathcal{C}$, where $f_{\mu}^{n}=f_{\mu}$ for every $n \in \mathbb{N}$, the functor $\underline{J}_{\mathcal{C}}:$ pro- $\mathcal{C} \rightarrow$ pro*-C is given.

For a given pair of categories $(\mathcal{C}, \mathcal{D})$, where $\mathcal{D}$ is dense in $\mathcal{C}$, the shape category $S h_{(\mathcal{C}, \mathcal{D})}$ has objects of $\mathcal{C}$ as objects, and its morphisms are $F=\langle\boldsymbol{f}\rangle=\left\langle\left[\left(f, f_{\mu}\right)\right]\right\rangle: X \rightarrow$ $Y$ for $\boldsymbol{f}=\left[\left(f, f_{\mu}\right)\right]: \boldsymbol{X} \rightarrow \boldsymbol{Y}$, where $\boldsymbol{X}$ and $\boldsymbol{Y}$ are systems associated with $X$ and $Y$ (respectively) by $\mathcal{D}$-expansions. Here, $\boldsymbol{f} \sim \boldsymbol{f}^{\prime}$ means that, if $\boldsymbol{i}: \boldsymbol{X} \rightarrow \boldsymbol{X}^{\prime}$ and $\boldsymbol{j}: \boldsymbol{Y} \rightarrow$ $\boldsymbol{Y}^{\prime}$ are canonical isomorphisms between expansions of the same object, $\boldsymbol{f}^{\prime} \boldsymbol{i}=\boldsymbol{j} \boldsymbol{f}$ in pro- $\mathcal{D}$. Obviously, the realizing category for the category $S h_{(\mathcal{C}, \mathcal{D})}$ is the category pro-D. Specially, the category HPol, the homotopy category of polyhedra, is dense in 
the category HTop, and the realizing category for the category $S h_{(H T o p, H P o l)} \equiv S h$ is the category pro-HPol.

For every $f: X \rightarrow Y$ of $\mathcal{C}$ and every pair of expansions $\boldsymbol{p}: X \rightarrow \boldsymbol{X}$ and $\boldsymbol{q}: Y \rightarrow \boldsymbol{Y}$ there exists a unique $\boldsymbol{f}: \boldsymbol{X} \rightarrow \boldsymbol{Y}$ of pro-D such that

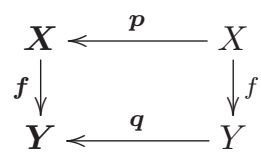

commutes in pro-C. The shape functor $S: \mathcal{C} \rightarrow S h_{(\mathcal{C}, \mathcal{D})}$ is defined by $S(X)=X, X \in$ $O b(\mathcal{C})$, and $S(f)=F=\langle\boldsymbol{f}\rangle, f \in \operatorname{Mor}(\mathcal{C})$.

\section{Reduced power of topological space}

Let $\mathcal{F}$ be a countable filter on $\mathbb{N}$ that consists of all $A \subseteq \mathbb{N}$ such that $\mathbb{N} \backslash A$ is finite. Let $X$ be a set. We will denote by $\tilde{X}$ the quotient set $\prod_{\mathbb{N}} \bar{X} / \sim$, where $\left(x_{n}\right) \sim\left(x_{n}^{\prime}\right)$ for $\left(x_{n}\right),\left(x_{n}^{\prime}\right) \in \prod_{\mathbb{N}} X$ if $\left\{n \mid x_{n}=x_{n}^{\prime}\right\} \in \mathcal{F}$.

We want to define a topology on $\tilde{X}$ when $(X, \mathcal{T})$ is a topological space. It is natural to take a quotient topology which we get from $\prod_{\mathbb{N}} X$ by projection. Let us show that the box topology on the cartesian product $\prod_{\mathbb{N}} X$ is, for our purpose, a better choice to introduce a topology on $\tilde{X}$ than the commonly used product topology.

Lemma 3.1. Let $(X, \mathcal{T})$ be a topological space, and let $\prod_{\mathbb{N}} X$ have the product topology $\mathcal{T}_{p}$. Then the quotient topology on $\tilde{X}$ is indiscrete.

Proof. A collection $\mathcal{B}_{p}=\left\{\prod_{\mathbb{N}} U_{n} \mid U_{n} \in \mathcal{T}, U_{n} \neq X\right.$ for only finitely many $\left.n\right\}$ is a basis for $\mathcal{T}_{p}$. Let $V \in \mathcal{B}_{p}$. Hence, $V=\prod_{\mathbb{N}} U_{n}$ where $U_{n} \in \mathcal{T}$ and $\left\{n \mid U_{n}=X\right\} \in \mathcal{F}$. Also, let $p: \prod_{\mathbb{N}} X \rightarrow \tilde{X}$ be a projection. Then

$$
\begin{aligned}
p(V)=p\left(\prod_{\mathbb{N}} U_{n}\right) & =\left\{\left[\left(x_{n}\right)\right] \in \tilde{X} \mid\left\{n \mid x_{n} \in U_{n}\right\} \in \mathcal{F}\right\} \\
& =\left\{\left[\left(x_{n}\right)\right] \in \tilde{X} \mid\left\{n \mid x_{n} \in X\right\} \in \mathcal{F}\right\}=\tilde{X} .
\end{aligned}
$$

If $U \subseteq \tilde{X}$ is open in $\tilde{X}, U \neq \emptyset$, then $p^{-1}(U)$ is open in $\prod_{\mathbb{N}} X$. Therefore, $p^{-1}(U)=$ $\bigcup_{V \in \mathcal{S}} V, \mathcal{S} \subseteq \mathcal{B}_{p}$. We have

$$
p\left(p^{-1}(U)\right)=p\left(\bigcup_{V \in \mathcal{S}} V\right)=\bigcup_{V \in \mathcal{S}} p(V)=\tilde{X} .
$$

Since $p\left(p^{-1}(U)\right) \subseteq U$, we get $\tilde{X} \subseteq U$, and consequently, $\tilde{X}=U$.

We will write $\underset{\mathbb{N}}{\nabla} U_{n} \equiv p\left(\prod_{\mathbb{N}} U_{n}\right)=\left\{\left[\left(x_{n}\right)\right] \in \tilde{X} \mid\left\{n \mid x_{n} \in U_{n}\right\} \in \mathcal{F}\right\}$ for $U_{n} \subseteq X$, $n \in \mathbb{N}$. 
Lemma 3.2. If the cartesian product $\prod_{\mathbb{N}} X$ is provided with the box topology $\mathcal{T}_{b}$, the collection

$$
\tilde{\mathcal{B}}=\left\{\underset{\mathbb{N}}{\nabla} U_{n} \mid U_{n} \in \mathcal{T}, \text { for every } n \in \mathbb{N}\right\}
$$

is a basis for the quotient topology on $\tilde{X}$.

Proof. Let us first show that $\tilde{\mathcal{B}}$ is indeed a topology basis. Let $\left[\left(x_{n}\right)\right] \in \tilde{X}$. Since $\mathcal{T}$ is a cover of $X$, for every $n \in \mathbb{N}$ there is $U_{n} \in \mathcal{T}$ such that $x_{n} \in U_{n}$. Thus, $\left[\left(x_{n}\right)\right] \in \underset{\mathbb{N}}{\nabla} U_{n}$, so $\tilde{\mathcal{B}}$ is a cover of $\tilde{X}$.

Let $\underset{\mathbb{N}}{\nabla} U_{n}, \nabla_{\mathbb{N}} V_{n} \in \tilde{\mathcal{B}}$. Then,

$$
\begin{aligned}
\underset{\mathbb{N}}{\nabla} U_{n} \cap \underset{\mathbb{N}}{\nabla} V_{n} & =\left\{\left[\left(x_{n}\right)\right] \in \tilde{X} \mid\left\{n \mid x_{n} \in U_{n}\right\} \in \mathcal{F}\right\} \cap\left\{\left[\left(x_{n}\right)\right] \in \tilde{X} \mid\left\{n \mid x_{n} \in V_{n}\right\} \in \mathcal{F}\right\} \\
& =\left\{\left[\left(x_{n}\right)\right] \in \tilde{X} \mid\left\{n \mid x_{n} \in U_{n} \cap V_{n}\right\} \in \mathcal{F}\right\} \\
& =\underset{\mathbb{N}}{\nabla}\left(U_{n} \cap V_{n}\right)
\end{aligned}
$$

implying that the intersection of any two elements of $\tilde{\mathcal{B}}$ is an element of $\tilde{\mathcal{B}}$.

Let us prove that $\tilde{\mathcal{B}}$ generates the quotient topology

$$
\tilde{\mathcal{T}}=\left\{U \subseteq \tilde{X} \mid p^{-1}(U) \subseteq \prod_{\mathbb{N}} X \text { is open in } \mathcal{T}_{b}\right\}
$$

Let $U \in \tilde{\mathcal{T}}$. That means, if $\mathcal{T}=\left\{U_{\lambda}, \lambda \in \Lambda\right\}$, that $p^{-1}(U)=\bigcup_{\alpha \in A} \prod_{\mathbb{N}} U_{\alpha_{n}}$ for some $A \subseteq$ $\Lambda^{\mathbb{N}}$ and $U_{\alpha_{n}} \in \mathcal{T}$ for every $\alpha \in A$. Since $p$ is a surjection, we have

$$
U=p\left(p^{-1}(U)\right)=p\left(\bigcup_{\alpha \in A} \prod_{\mathbb{N}} U_{\alpha_{n}}\right)=\bigcup_{\alpha \in A} p\left(\prod_{\mathbb{N}} U_{\alpha_{n}}\right)=\bigcup_{\alpha \in A} \underset{\mathbb{N}}{\nabla} U_{\alpha_{n}} .
$$

Remark 3.3. Usually, when a product space $\prod_{\mathbb{N}} X$ is provided with the box topology, it is denoted by $\square_{\mathbb{N}} X$, and when it is provided with the product topology, it is denoted by $X^{\omega}$.

The space $(\tilde{X}, \tilde{\mathcal{T}})$, abbreviated $\tilde{X}$, can be found in studies of Rudin, Kunen and Bankston (for example in $[\mathbf{6}, \mathbf{3}]$ or $[\mathbf{1}]$ ), and is referred to as a reduced box product or a reduced power. In the sequel we will list some properties of this space, and we will include some simple concise proofs with new denotation.

Theorem 3.4. If $X$ is a discrete space, then $\tilde{X}$ is a discrete space, too.

Proof. The statement is obvious.

Lemma 3.5. If $\left(F_{n}\right)$ is a sequence of sets such that $F_{n} \subseteq X$ is closed in $X$ for every $n \in \mathbb{N}$, then $\underset{\mathbb{N}}{\nabla} F_{n}$ is closed in $\tilde{X}$. 
Proof. The complement of $\underset{\mathbb{N}}{\nabla} F_{n}$ in $\tilde{X}$ is given with

$$
\left(\nabla_{\mathbb{N}} F_{n}\right)^{c}=\left\{\left[\left(x_{n}\right)\right] \mid\left\{n \mid x_{n} \in F_{n}\right\} \notin \mathcal{F}\right\}=\left\{\left[\left(x_{n}\right)\right] \mid\left\{n \mid x_{n} \in F_{n}^{c}\right\} \text { is cofinal in } \mathbb{N}\right\} .
$$

Therefore, its preimage by the projection $p$ is

$$
p^{-1}\left(\left(\nabla_{\mathbb{N}} F_{n}\right)^{c}\right)=\bigcup_{\substack{K \subseteq \mathbb{N} \\
\text { cofinal in } \mathbb{N}}} \prod_{\mathbb{N}} W_{K n}, \quad \text { where } \quad W_{K n}=\left\{\begin{array}{cl}
F_{n}^{c}, & n \in K \\
X, & n \in \mathbb{N} \backslash K
\end{array}\right.
$$

and that is an open set in $\underset{\mathbb{N}}{\square}$.

Theorem 3.6. If $X$ is a $T_{i}$ space for $i=0,1,2$ or 3 , then $\tilde{X}$ is also a $T_{i}$ space.

Proof. We will provide the proof of the statement for $T_{1}$ and $T_{3}$ spaces. The rest is similar.

Let $(X, \mathcal{T})$ be a $T_{1}$ space and let $\left[\left(x_{n}\right)\right],\left[\left(y_{n}\right)\right] \in \tilde{X},\left[\left(x_{n}\right)\right] \neq=\left[\left(y_{n}\right)\right]$. That means there is an infinite set $A \subseteq \mathbb{N}$ such that $x_{n} \neq y_{n}$ for every $n \in A$. $X$ is a $T_{1}$ space, so, for every $n \in A$ there is an open neighborhood $U_{n} \subseteq X$ of $x_{n}$ which does not contain $y_{n}$. For $n \in \mathbb{N} \backslash A$ let $U_{n}=X$. In this way we get a neighborhood $\nabla_{\mathbb{N}} U_{n}$ of $\left[\left(x_{n}\right)\right]$ that does not contain $\left[\left(y_{n}\right)\right]$, implying that $(\tilde{X}, \tilde{\mathcal{T}})$ is a $T_{1}$ space.

Now, let $(X, \mathcal{T})$ be a regular space. According to the previous, $\tilde{X}$ is a $T_{1}$ space. For arbitrarily chosen $\left[\left(x_{n}\right)\right] \in \tilde{X}$ and $F \subseteq \tilde{X}$ closed in $\tilde{X}$ such that $\left[\left(x_{n}\right)\right] \notin F$, there is a basic set $\underset{\mathbb{N}}{\nabla} U_{n}$ such that $\left[\left(x_{n}\right)\right] \in \underset{\mathbb{N}}{\nabla} U_{n}$, and $F \subseteq \tilde{X} \backslash \nabla_{\mathbb{N}} U_{n}$. Then $J:=\left\{n \mid x_{n} \in U_{n}\right\} \in$ $\mathcal{F}$. Since $X$ is regular, for every $n \in J$ we can choose an open set $V_{n} \subseteq X$ such that

$$
x_{n} \in V_{n} \subseteq \bar{V}_{n} \subseteq U_{n} .
$$

For $n \notin J$ let us put $V_{n}=X$. We have

$$
\left[\left(x_{n}\right)\right] \in \underset{\mathbb{N}}{\nabla} V_{n} \subseteq \underset{\mathbb{N}}{\nabla} \bar{V}_{n} \subseteq \underset{\mathbb{N}}{\nabla} U_{n}
$$

According to 3.5 , the set $\underset{\mathbb{N}}{\nabla} \bar{V}_{n}$ is closed in $\tilde{X}$. So, $\tilde{X} \backslash \underset{\mathbb{N}}{\nabla} \bar{V}_{n}$ is an open set in $\tilde{X}$ containing $F$, and $\underset{\mathbb{N}}{\nabla} V_{n} \cap\left(\tilde{X} \backslash \nabla_{\mathbb{N}} \bar{V}_{n}\right)=\emptyset$.

The next statement can be found in $[\mathbf{6}]$ and $[\mathbf{3}]$ in a more general form.

Theorem 3.7. $\tilde{X}$ is a P-space.

Proof. We need to prove that an intersection of a countable collection of open sets in $\tilde{X}$ is an open set. It is sufficient to prove the statement for basic sets.

Let $\left[\left(x_{n}\right)\right] \in \bigcap_{k \in \mathbb{N}} \nabla_{\mathbb{N}} U_{n}^{k}$, where $U_{n}^{k} \in \mathcal{T}$ for every $k \in \mathbb{N}$ and for every $n \in \mathbb{N}$. Let $F(n)=\left\{k \in \mathbb{N} \mid k \leqslant n, x_{n} \in U_{n}^{k}\right\}$. The set $F(n)$ is finite, and therefore

$$
V_{n}=\bigcap_{k \in F(n)} U_{n}^{k}
$$

is open in $X$. Notice that $F(n)$ can be empty for a finite number of $n \in \mathbb{N}$. In that case, let $V_{n}$ be the whole space $X$. We claim that $\left[\left(x_{n}\right)\right] \in \nabla_{\mathbb{N}} V_{n} \subseteq \bigcap_{k \in \mathbb{N}} \nabla_{\mathbb{N}} U_{n}^{k}$. If we fix 
some $k \in \mathbb{N}$, we have

$$
\{n \mid k \in F(n)\}=\left\{n \mid k \leqslant n, x_{n} \in U_{n}^{k}\right\} \in \mathcal{F} .
$$

Also,

$$
\{n \mid k \in F(n)\} \subseteq\left\{n \mid V_{n} \subseteq U_{n}^{k}\right\} \in \mathcal{F},
$$

which implies

$$
\left[\left(x_{n}\right)\right] \in \underset{\mathbb{N}}{\nabla} V_{n} \subseteq \underset{\mathbb{N}}{\nabla} U_{n}^{k},
$$

for arbitrary $k \in \mathbb{N}$. Therefore,

$$
\left[\left(x_{n}\right)\right] \in \underset{\mathbb{N}}{\nabla} V_{n} \subseteq \bigcap_{k \in \mathbb{N}} \nabla_{\mathbb{N}} U_{n}^{k}
$$

and we conclude that $\bigcap_{k \in \mathbb{N}} \nabla_{\mathbb{N}} U_{n}^{k}$ is open in $\tilde{X}$.

Remark 3.8. There are several definitions of a $P$-space in the literature. We use the definition in the sense of Gillman-Henriksen.

According to [5], in a $P$-space, regularity and complete regularity are equivalent. It can be easily seen from the fact that in a regular space $X$ for every $x \in X$ and $F \subseteq X$ closed in $X$ not containing $x$ we can construct a sequence $\left(V_{n}\right)$ of open sets in $X$ such that

$$
x \in V_{n} \subseteq X \backslash F, \text { for every } n \in \mathbb{N}
$$

and

$$
\bar{V}_{n+1} \subseteq V_{n}, \text { for every } n \in \mathbb{N} .
$$

Then, $x \in \bigcap_{n \in \mathbb{N}} V_{n}$, and if $X$ is a $P$-space, the set $\bigcap_{n \in \mathbb{N}} V_{n}$ is open in $X$. It is also closed since $\bigcap_{n \in \mathbb{N}} V_{n}=\bigcap_{n \in \mathbb{N}} \bar{V}_{n}$, and it is disjoint with $F$, so the map $f: X \rightarrow[0,1]$ defined by

$$
f(x)= \begin{cases}1, & x \in \bigcap_{n \in \mathbb{N}} V_{n}, \\ 0, & x \in X \backslash \bigcap_{n \in \mathbb{N}} V_{n},\end{cases}
$$

is continuous. Obviously, every regular $P$-space has a clopen base. Consequently, every regular $P$-space is totally disconnected (every point in such a space can be separated from every other point by a clopen neighborhood).

Hence, if $X$ is a completely regular space, $\tilde{X}$ is a completely regular space, too, and it is totally disconnected.

Also, if $X$ is zero-dimensional with respect to the small inductive dimension, or totally disconnected, so is $\tilde{X}$ (see $[\mathbf{1}]$ ).

Example 3.9. The space $\square_{\mathbb{N}}\{0,1\} / \sim$ is discrete and it is an example of a reduced power of a compact space that is not compact.

Example 3.10. An example of a $P$-space that is not discrete is a reduced power of Sierpiński space. If $X=\{0,1\}$ has a topology $\mathcal{T}=\{\emptyset,\{1\},\{0,1\}\}$, then $\left\{\left[\left(x_{n}\right)\right]\right\}$ is not open in $\tilde{X}$ whenever $\left\{n \in \mathbb{N} \mid x_{n}=1\right\} \notin \mathcal{F}$. 
Example 3.11. The space $\tilde{\mathbb{R}}$ is completely regular since $\mathbb{R}$ is, and it is zero-dimensional and totally disconnected.

More properties of $\tilde{X}$ can be found in [5] and [6].

\section{Box-homotopy}

To clear the matter of continuity of mappings that we use in the sequel, we begin with simple lemmata.

Lemma 4.1. The projection $p_{n}: \square_{\mathbb{N}} X \rightarrow X$ to the $n$-th coordinate space is a continuous mapping, for every $n \in \mathbb{N}$.

Proof. The statement is obviously true.

Remark 4.2. The similar statement for the product topology is an elementary fact.

Lemma 4.3. Let $X_{n}$ and $Y_{n}$ be topological spaces and $f_{n}: X_{n} \rightarrow Y_{n}$ a mapping, for every $n \in \mathbb{N}$. The mapping $\prod_{\mathbb{N}} f_{n}: \prod_{\mathbb{N}} X_{n} \rightarrow \prod_{\mathbb{N}} Y_{n}$ between products, both with either product topologies or box topologies, defined by

$$
\prod_{\mathbb{N}} f_{n}\left(\left(x_{n}\right)\right)=\left(f_{n}\left(x_{n}\right)\right)
$$

is continuous if and only if $f_{n}$ is continuous for every $n$.

Proof. Let us prove the statement in the case of box topology on products. The product topology case is an elementary fact.

Let $\square V_{\mathbb{N}} V_{n}$ be a basic set in $\square Y_{n}$. That means that $V_{n}$ is open in $Y_{n}$ for every $n \in \mathbb{N}$. We have

$$
\left(\prod_{\mathbb{N}} f_{n}\right)^{-1}\left(\square_{\mathbb{N}} V_{n}\right)=\square_{\mathbb{N}}\left(\left(f_{n}\right)^{-1}\left(V_{n}\right)\right) .
$$

If $f_{n}$ is continuous for every $n \in \mathbb{N},\left(f_{n}\right)^{-1}\left(V_{n}\right)$ is open in $X_{n}$ for every $n \in \mathbb{N}$, hence $\square_{\mathbb{N}}\left(\left(f_{n}\right)^{-1}\left(V_{n}\right)\right)$ is open in $\underset{\mathbb{N}}{\square} X_{n}$, implying that $\prod_{\mathbb{N}} f_{n}$ is continuous.

To prove the converse, let us fix any $k \in \mathbb{N}$, let $V_{k} \subset Y_{k}$ be an open set in $Y_{k}$ and let $V_{n}=Y_{n}$ for $n \neq k$. Then,

$$
\left(\prod_{\mathbb{N}} f_{n}\right)^{-1}\left(\square_{\mathbb{N}} V_{n}\right)=\square_{\mathbb{N}} W_{n},
$$

where $W_{n}=X_{n}$ for $n \neq k$ and $W_{k}=f_{k}^{-1}\left(V_{k}\right)$. If $\prod_{\mathbb{N}} f_{n}$ is continuous, then $\underset{\mathbb{N}}{\square} W_{n}$ is open in $\underset{\mathbb{N}}{\square_{n}} X_{n}$. Hence, $W_{k}$ is open in $X_{k}$, implying that $f_{k}$ is continuous. Since $k$ was arbitrarily chosen, we conclude that $f_{n}$ is continuous for every $n \in \mathbb{N}$.

Remark 4.4. The statement of the previous lemma is obviously valid if we replace sequences with finite (of course, equal) number of spaces and mappings. 
Definition 4.5. Let $X, Y$ be topological spaces, and $f, g: X \rightarrow Y$ continuous maps, and let $n \in \mathbb{N} \cup\{\omega\}$. We will say that $f$ is $n$-homotopic to $g, f \sim r$, if there is a continuous mapping $H: X \times I^{n} \rightarrow Y$, where $I^{n}$ has the product topology, satisfying

$$
\begin{aligned}
& H(x, 0,0,0, \ldots, 0)=f(x), \\
& H(x, 1,1,1, \ldots, 1)=g(x),
\end{aligned}
$$

when $n \in \mathbb{N}$, and

$$
\begin{aligned}
& H(x, 0,0,0, \ldots)=f(x), \\
& H(x, 1,1,1, \ldots)=g(x),
\end{aligned}
$$

for $n=\omega$, for every $x \in X$.

Proposition 4.6. Let $n \in \mathbb{N} \cup\{\omega\}$ and let $f, g \in T o p(X, Y)$. Then $f$ is $n$-homotopic to $g$ if and only if $f$ is homotopic to $g$.

Proof. The statement of the theorem is obvious if $n=1(\sim=\simeq)$.

Let us fix an $n \in \mathbb{N} \cup\{\omega\}$. If $H: X \times I \rightarrow Y$ is a homotopy from $f$ to $g$, then the mapping $H_{n}: X \times I^{n} \rightarrow Y$ defined by $H_{n}=H \circ\left(\operatorname{id}_{X} \times p_{1}\right)$, where $p_{1}: I^{n} \rightarrow I$ is a projection to the first coordinate, is an $n$-homotopy from $f$ to $g$. It is continuous because projection is continuous.

Likewise, if $H_{n}: X \times I^{n} \rightarrow Y$ is an $n$-homotopy form $f$ to $g$, then $H: X \times I \rightarrow Y$ defined by $H=H_{n} \circ\left(\mathrm{id}_{X} \times \Delta_{I}\right)$, where $\Delta_{I}: I \rightarrow I^{n}$ is defined by $\Delta_{I}(t)=(t, t, \ldots)$, is a homotopy from $f$ to $g$. It is continuous for every $n \in \mathbb{N} \cup\{\omega\}$ because of the universal property for the product topology saying that a mapping to the product of an arbitrary collection of topological spaces (with the product topology) is continuous if and only if all its coordinate mappings are continuous.

To generalize the notion of homotopy, we give the following definition.

Definition 4.7. Let $X, Y$ be topological spaces, and $f, g: X \rightarrow Y$ continuous mappings. We will say that $f$ and $g$ are box-homotopic, $f \sim g$, if there is a continuous mapping $H: X \times \square_{\mathbb{N}} I \rightarrow Y$ satisfying

$$
\begin{aligned}
& H(x, 0,0,0, \ldots)=f(x), \\
& H(x, 1,1,1, \ldots)=g(x),
\end{aligned}
$$

for every $x \in X$.

To prove that the box-homotopy is an equivalence relation on $\operatorname{Top}(X, Y)$, we will need the following lemma.

Lemma 4.8. $\triangle=\{(x, x, x, \ldots) \mid x \in I\} \subset \square_{\mathbb{N}} I$ is a retract of the space $\square_{\mathbb{N}} I$.

Proof. Let

$$
\begin{aligned}
& C=\left\{\left(x_{n}\right) \in \underset{\mathbb{N}}{\square} \mid\left(x_{n}\right) \text { converges in } I\right\} \subset \underset{\mathbb{N}}{\square} I \\
& D=\left\{\left(x_{n}\right) \in \underset{\mathbb{N}}{\square} \mid\left(x_{n}\right) \text { diverges in } I\right\} \subset \square_{\mathbb{N}} I .
\end{aligned}
$$


$C$ is open since every $\left(x_{n}\right) \in C$ has an open neighborhood

$$
\square_{\mathbb{N}}\left(\left\langle x_{n}-\frac{1}{2^{n}}, x_{n}+\frac{1}{2^{n}}\right\rangle \cap I\right) \subset C .
$$

$D$ is also open since every $\left(x_{n}\right) \in D$ has an open neighborhood

$$
\square_{\mathbb{N}}\left(\left\langle x_{n}-\frac{1}{2^{n}}, x_{n}+\frac{1}{2^{n}}\right\rangle \cap I\right) \subset D .
$$

Therefore, $\{C, D\}$ is a separation of $\underset{\mathbb{N}}{\square}$.

We claim that a mapping $f: C \rightarrow \square_{\mathbb{N}} I$ defined by $f\left(\left(x_{n}\right)\right)=(x, x, \ldots)$, where $x=$ $\lim _{n \rightarrow \infty}\left(x_{n}\right)$, is continuous. Let $\underset{\mathbb{N}}{\square} V_{n} \subset \underset{\mathbb{N}}{\square} I$ be an arbitrary basic set. If $f^{-1}\left(\underset{\mathbb{N}}{\square} V_{n}\right) \neq \emptyset$, and $\left(x_{n}\right) \in f^{-1}\left(\underset{\mathbb{N}}{\square} V_{n}\right)$ with $x=\lim _{n \rightarrow \infty}\left(x_{n}\right)$, then

$$
f\left(\left(\square\left\langle x_{n}-\frac{1}{2^{n}}, x_{n}+\frac{1}{2^{n}}\right\rangle\right) \cap \square_{\mathbb{N}} I\right)=\{(x, x, x, \ldots)\} \subset \square_{\mathbb{N}} V_{n} .
$$

Therefore, $f$ is continuous.

Now, we can define $r: \underset{\mathbb{N}}{\square} I \rightarrow \triangle$ by

$$
r\left(\left(x_{n}\right)\right)=\left\{\begin{array}{ll}
\left(\frac{1}{2}, \frac{1}{2}, \ldots\right) & \left(x_{n}\right) \in D \\
f\left(\left(x_{n}\right)\right) & \left(x_{n}\right) \in C
\end{array} .\right.
$$

Since $\{C, D\}$ is a separation of $\square \mathbb{N} I, r$ is continuous. Also, $r / \triangle=\mathrm{id}_{\triangle}$. Therefore, $r$ is a retraction from $\square_{\mathbb{N}} I$ to its subspace $\triangle$.

Theorem 4.9. To be box-homotopic is an equivalence relation on Top $(X, Y)$.

Proof. Reflexivity. The mapping $H: X \times \underset{\mathbb{N}}{\square} I \rightarrow Y$ defined by $H=f \circ p_{1}$ is continuous according to 4.1, and it is a box-homotopy from $f$ to $f$.

Symmetry. If $H: X \times \square_{\mathbb{N}} I \rightarrow Y$ is a box-homotopy from $f$ to $g$, then the map $G: X \times \underset{\mathbb{N}}{\square} I \rightarrow Y, G=H \circ\left(\mathrm{id}_{X} \times\left(1-\mathrm{id}_{I}\right) \times\left(1-\mathrm{id}_{I}\right) \times \cdots\right)$, is a box-homotopy from $g$ to $f$. According to 4.3 it is continuous.

Transitivity. Suppose $H_{1}: X \times \square_{\mathbb{N}} I \rightarrow Y$ is a box-homotopy from $f$ to $g$, and $H_{2}: X \times$ $\square_{\mathbb{N}} I \rightarrow Y$ is a box homotopy from $g$ to $h$. The mapping $H: X \times\left(\prod_{\mathbb{N}}\left[0, \frac{1}{2}\right] \cup \prod_{\mathbb{N}}\left[\frac{1}{2}, 1\right]\right) \rightarrow$ $Y$, where $\prod_{\mathbb{N}}\left[0, \frac{1}{2}\right] \cup \prod_{\mathbb{N}}\left[\frac{1}{2}, 1\right] \subset \square_{\mathbb{N}} I$ has the subspace topology, defined by

$$
\begin{aligned}
& H /_{X \times \prod_{\mathbb{N}}\left[0, \frac{1}{2}\right]}=H_{1} \circ\left(\operatorname{id}_{X} \times\left(2 \cdot \operatorname{id}_{\left[0, \frac{1}{2}\right]}\right) \times\left(2 \cdot \operatorname{id}_{\left[0, \frac{1}{2}\right]}\right) \times \cdots\right), \\
& H /_{X \times \prod_{\mathbb{N}}\left[\frac{1}{2}, 1\right]}=H_{2} \circ\left(\operatorname{id}_{X} \times\left(2 \cdot \operatorname{id}_{\left[\frac{1}{2}, 1\right]}-1\right) \times\left(2 \cdot \operatorname{id}_{\left[\frac{1}{2}, 1\right]}-1\right) \times \cdots\right)
\end{aligned}
$$

is continuous according to 4.3 and the pasting lemma because $X \times \prod_{\mathbb{N}}\left[0, \frac{1}{2}\right]$ and $X \times$ $\prod_{\mathbb{N}}\left[\frac{1}{2}, 1\right]$ are closed in $X \times\left(\prod_{\mathbb{N}}\left[0, \frac{1}{2}\right] \cup \prod_{\mathbb{N}}\left[\frac{1}{2}, 1\right]\right)$, and $H / X \times \prod_{\mathbb{N}}\left[0, \frac{1}{2}\right]$ and $H / X \times \prod_{\mathbb{N}}\left[\frac{1}{2}, 1\right]$ 
coincide on their intersection $X \times\left\{\left(\frac{1}{2}, \frac{1}{2}, \frac{1}{2}, \ldots\right)\right\}$. Also,

$$
\begin{aligned}
& H(x, 0,0, \ldots)=H_{1}(x, 0,0, \ldots)=f(x), \\
& H(x, 1,1, \ldots)=H_{2}(x, 1,1, \ldots)=h(x) .
\end{aligned}
$$

Hence, if $r$ is a retraction from $\square_{\mathbb{N}} I$ to its subspace $\triangle$, the mapping $H \circ\left(\operatorname{id}_{X} \times r\right)$ is a box-homotopy from $f$ to $h$.

Theorem 4.10. Let $f, f^{\prime}: X \rightarrow Y$ and $g, g^{\prime}: Y \rightarrow Z$ be continuous maps, and let $g \circ f, g^{\prime} \circ f^{\prime}: X \rightarrow Z$ be the respective composite maps. If $f \sim f^{\prime}$ and $g \sim g^{\prime}$, then $g \circ f \tilde{\square} g^{\prime} \circ f^{\prime}$.

Proof. Let $H_{1}: X \times \underset{\mathbb{N}}{\square} \rightarrow Y$ be a box-homotopy from $f$ to $f^{\prime}$, and $H_{2}: Y \times \underset{\mathbb{N}}{\square} I \rightarrow$ $Z$ a box homotopy from $g$ to $g^{\prime}$. The map $G_{1}=g \circ H_{1}: X \times \underset{\mathbb{N}}{\square} \rightarrow Z$ is a boxhomotopy from $g \circ f$ to $g \circ f^{\prime}$, and the map $G_{2}: X \times \square_{\mathbb{N}} I \rightarrow Z$ defined by $G_{2}=$ $H_{2} \circ\left(\left(f^{\prime} \circ \mathrm{id}_{X}\right) \times \mathrm{id}_{I} \times \mathrm{id}_{I} \times \cdots\right)$ is a box-homotopy from $g \circ f^{\prime}$ to $g^{\prime} \circ f^{\prime}$. Now, using transitivity of the relation $\underset{\square}{\sim}$, we conclude that $g \circ f \underset{\square}{\sim} g^{\prime} \circ f^{\prime}$.

Theorem 4.11. If two mappings are homotopic, then they are box-homotopic.

Proof. Let $H: X \times I \rightarrow Y$ be a homotopy from $f$ to $g$. We can define a box-homotopy $G: X \times \underset{\mathbb{N}}{\square} I \rightarrow Y$ from $f$ to $g$ by $G=H \circ\left(\operatorname{id}_{X} \times p_{1}\right)$.

Remark 4.12. The converse does not hold due to the fact that the universal property of product topology does not have its equivalent for the box topology. We will provide a counterexample in the sequel.

The relation $\sim$ divides the set $\operatorname{Mor}(T o p)$ into equivalence classes, and it is a congruence (it is well adjusted with the composition). Therefore, there exists the quotient category $T o p / \sim$. We will denote it by $H_{\square}$ Top, and the box-homotopy class of a morphism $f$ will be denoted by $[f]_{\square}$.

\section{The reduction of $p r o^{*}-H T o p$ category}

If a morphism $\left[\left(f^{n}\right)\right]:(X) \rightarrow(Y)$ between rudimentary systems in pro*-Top is given, we will denote by $\underset{\mathbb{N}}{\nabla} f^{n}$ the mapping from $(\tilde{X})$ to $(\tilde{Y})$ induced by $\prod_{\mathbb{N}} f^{n}: \underset{\mathbb{N}}{\square} X \rightarrow$ $\square_{\mathbb{N}} Y, \prod_{\mathbb{N}} f^{n}\left(\left(x_{n}\right)\right)=\left(f^{n}\left(x_{n}\right)\right)$, for $\left(x_{n}\right) \in \square_{\mathbb{N}} X$.

Theorem 5.1. The mapping $\underset{\mathbb{N}}{\nabla} f^{n}$ is a well defined morphism of pro-Top.

Proof. Let $\left(f^{n}\right) \in i n v^{*}-T o p((X),(Y))$. The map $\prod_{\mathbb{N}} f^{n}: \underset{\mathbb{N}}{\square} X \rightarrow \underset{\mathbb{N}}{\square} Y$ is continuous according to 4.3. Suppose $p: \underset{\mathbb{N}}{\square} X \rightarrow \tilde{X}$ and $q: \underset{\mathbb{N}}{\square} Y \rightarrow \tilde{Y}$ are quotient maps. Then, 
$q\left(\prod_{\mathbb{N}} f^{n}\right): \square_{\mathbb{N}} X \rightarrow \tilde{Y}$ is continuous and, for $\left(x_{n}\right),\left(x_{n}^{\prime}\right) \in \square_{\mathbb{N}} X$ such that $\left(x_{n}\right) \sim\left(x_{n}^{\prime}\right)$,

$$
\left(q\left(\prod_{\mathbb{N}} f^{n}\right)\right)\left(\left(x_{n}\right)\right)=\left(q\left(\prod_{\mathbb{N}} f^{n}\right)\right)\left(\left(x_{n}^{\prime}\right)\right) .
$$

Thus there is a unique continuous map $g: \tilde{X} \rightarrow \tilde{Y}$ such that

$$
g \circ p=q \circ\left(\prod_{\mathbb{N}} f^{n}\right) .
$$

Obviously, $g=\nabla_{\mathbb{N}} f^{n}$. Let $\left(f^{n}\right),\left(f^{\prime n}\right) \in i n v^{*}-T o p((X),(Y))$ such that $f^{n}=f^{\prime n}$ for all but finitely many $n \in \mathbb{N}$. Then $\left(\prod_{\mathbb{N}} f^{n}\right)\left(\left(x_{n}\right)\right) \sim\left(\prod_{\mathbb{N}} f^{\prime n}\right)\left(\left(x_{n}\right)\right)$ in $\square_{\mathbb{N}} Y$ for $\left(x_{n}\right) \in$ $\underset{\mathbb{N}}{\square} X$. Hence, $\prod_{\mathbb{N}} f^{n}$ and $\prod_{\mathbb{N}} f^{\prime n}$ induce the same map $\underset{\mathbb{N}}{\nabla} f^{n}$.

Lemma 5.2. Let $X$ and $Y$ be two topological spaces and let $p_{n}: \underset{\mathbb{N}}{\square} X \rightarrow X$ and $q_{n}: \underset{\mathbb{N}}{\square} \rightarrow Y$ be projections to the $n$-th coordinate space, for every $n \in \mathbb{N}$. Then, the mapping

$$
i=\left(p_{1} \times q_{1}, p_{2} \times q_{2}, \ldots\right):(\square X) \times\left(\square_{\mathbb{N}} X\right) \rightarrow \underset{\mathbb{N}}{\square}(X \times Y)
$$

is a homeomorphism.

Proof. The mapping $i$ is obviously a bijection. A basic open set in $\underset{\mathbb{N}}{\square}(X \times Y)$ is of the form $\underset{\mathbb{N}}{\square}\left(U_{n} \times V_{n}\right)$, where $U_{n} \subset X$ is open in $X$ and $V_{n} \subset Y$ is open in $Y$, for every $n \in \mathbb{N}$. Its preimage under $i$ is

$$
\begin{aligned}
\left(\left(p_{1} \times q_{1}\right)^{-1}\left(U_{1} \times V_{1}\right)\right) \cap\left(\left(p_{2} \times q_{2}\right)^{-1}\left(U_{2} \times V_{2}\right)\right) \cap \cdots \\
=\left(\left(p_{1}\right)^{-1}\left(U_{1}\right) \times\left(q_{1}\right)^{-1}\left(V_{1}\right)\right) \cap\left(\left(p_{2}\right)^{-1}\left(U_{2}\right) \times\left(q_{2}\right)^{-1}\left(V_{2}\right)\right) \cap \cdots \\
=\left(\bigcap_{\mathbb{N}}\left(p_{n}\right)^{-1}\left(U_{n}\right)\right) \times\left(\bigcap_{\mathbb{N}}\left(q_{n}\right)^{-1}\left(V_{n}\right)\right) \\
=\left(\square_{\mathbb{N}} U_{n}\right) \times\left(\square_{\mathbb{N}} V_{n}\right),
\end{aligned}
$$

and that is an open set in $(\underset{\mathbb{N}}{\square} X) \times(\underset{\mathbb{N}}{\square} Y)$. Therefore, $i$ is continuous. Moreover, since

$$
i\left(\left(\square U_{n}\right) \times\left(\square \square_{\mathbb{N}} V_{n}\right)\right)=\underset{\mathbb{N}}{\square}\left(U_{n} \times V_{n}\right),
$$

$i^{-1}$ is continuous as well.

Theorem 5.3. Let $\left(f^{n}\right),\left(f^{\prime n}\right) \in i n v^{*}-T o p((X),(Y))$ such that $f^{n}$ is homotopic to $f^{\prime n}$ for every $n \in \mathbb{N}$. Then $\nabla_{\mathbb{N}} f^{n} \sim \underset{\mathbb{N}}{\nabla} f^{\prime n}$.

Proof. Let, for every $n \in \mathbb{N}$, a map $H_{n}: X \times I \rightarrow Y$ be a homotopy from $f^{n}$ to $f^{\prime n}$. Let $i$ be the homeomorphism from Lemma 5.2. The mapping $H: \square_{\mathbb{N}} X \times \underset{\mathbb{N}}{\square} I \rightarrow \underset{\mathbb{N}}{\square} Y$ defined by $H=\left(\prod_{\mathbb{N}} H_{n}\right) \circ i$ is continuous by Lemmas 4.3 and 5.2 , and it induces 
a continuous map $\tilde{H}: \tilde{X} \times \underset{\mathbb{N}}{\square} I \rightarrow \tilde{Y}$. Indeed, for any fixed $\left(t_{n}\right) \in \underset{\mathbb{N}}{\square} I$, if $\left(x_{n}\right) \sim\left(x_{n}^{\prime}\right)$, then

$$
\left\{n \mid H_{n}\left(x_{n}, t_{n}\right)=H_{n}\left(x_{n}^{\prime}, t_{n}\right)\right\} \in \mathcal{F} .
$$

Thus $\left(H_{n}\left(x_{n}, t_{n}\right)\right) \sim\left(H_{n}\left(x_{n}^{\prime}, t_{n}\right)\right)$. It is easy to verify that $\tilde{H} / \tilde{X} \times\{(0,0, \ldots)\}=\underset{\mathbb{N}}{\nabla} f^{n}$ and $\tilde{H} / \tilde{X} \times\{(1,1, \ldots)\}=\underset{\mathbb{N}}{\nabla} f^{\prime n}$.

Lemma 5.4. The mapping $j: \underset{\mathbb{N}}{\square} I \rightarrow \square_{\mathbb{N}}\left(\square_{\mathbb{N}} I\right)$ defined by

$$
j\left(\left(x_{n}\right)\right)=\left(\left(y_{n}^{1}\right),\left(y_{n}^{2}\right), \ldots\right),
$$

where $y_{n}^{m}=x_{2^{m-1}(2 n-1)}$ for every $m, n \in \mathbb{N}$, is a homeomorphism.

Proof. Notice that the mapping $c: \mathbb{N} \times \mathbb{N} \rightarrow \mathbb{N}, c(m, n)=2^{m-1}(2 n-1)$ is a bijection commonly used for proving that the Cartesian product of two countable sets is countable. Therefore, $j$ is a bijection.

Let $\underset{m \in \mathbb{N}}{\square}\left(\underset{n \in \mathbb{N}}{\square} V_{n}^{m}\right)$ be a basic open set in $\square_{\mathbb{N}}(\underset{\mathbb{N}}{\square} X)$.

$$
j_{X}^{-1}\left(\underset{m \in \mathbb{N}}{\square}\left(\underset{n \in \mathbb{N}}{\square} V_{n}^{m}\right)\right)=j_{X}^{-1}\left(\underset{n \in \mathbb{N}}{\square} V_{n}^{1} \times \underset{n \in \mathbb{N}}{\square} V_{n}^{2} \times \cdots\right)=\underset{k \in \mathbb{N}}{\square} U_{k},
$$

where $U_{k}=V_{n}^{m}$ for $k=c(m, n)$. Therefore, $j_{X}$ is continuous.

The openness of $j_{X}$ is proved analogously.

Remark 5.5. The similar statement is valid if $I$ is replaced by $X$, for every $X \in$ $O b(T o p)$.

Theorem 5.6. Let $\left(f^{n}\right),\left(f^{\prime n}\right) \in i n v^{*}-T o p((X),(Y))$ such that $f^{n}$ is box-homotopic to $f^{\prime n}$ for every $n \in \mathbb{N}$. Then $\underset{\mathbb{N}}{\nabla} f^{n} \sim \nabla_{\mathbb{N}} f^{\prime n}$.

Proof. Let, for every $n \in \mathbb{N}$, a map $H_{n}: X \times \square_{\mathbb{N}} I \rightarrow Y$ be a box-homotopy from $f^{n}$ to $f^{\prime n}$. Let $i$ and $j_{I}$ be the mappings from Lemmas 5.2 and 5.4. The mapping $H: \underset{\mathbb{N}}{\square} \times$ $\square_{\mathbb{N}} I \rightarrow \underset{\mathbb{N}}{\square} Y$ defined by

$$
H=\left(\prod_{\mathbb{N}} H_{n}\right) \circ i \circ\left(\operatorname{id}_{\square X} \times j_{I}\right)
$$

is continuous by Lemmas 4.3, 5.2 and 5.4, therefore it is a box-homotopy between $\prod_{\mathbb{N}} f^{n}$ and $\prod_{\mathbb{N}} f^{\prime n}$, and it induces a continuous map $\tilde{H}: \tilde{X} \times \underset{\mathbb{N}}{\square} I \rightarrow \tilde{Y}$, a box-homotopy between $\underset{\mathbb{N}}{\nabla} f^{n}$ and $\underset{\mathbb{N}}{\nabla} f^{\prime n}$.

The following simple example shows that box-homotopic maps need not be homotopic.

Example 5.7. Let $\left(f^{n}\right),\left(g^{n}\right) \in i n v^{*}-T o p((I),(I))$ such that $f^{n}=0$ and $g^{n}=1$ for every $n \in \mathbb{N}$. The maps $f^{n}$ and $g^{n}$ are homotopic for every $n \in \mathbb{N}$, and, as we have seen, $\nabla_{\mathbb{N}} f^{n}, \underset{\mathbb{N}}{\nabla} g^{n}: \tilde{I} \rightarrow \tilde{I}$ are box-homotopic by Theorem 5.3. But, they are not homotopic. 
Indeed, if $H: \tilde{I} \times I \rightarrow \tilde{I}$ is a homotopy between $\underset{\mathbb{N}}{\nabla} f^{n}$ and $\underset{\mathbb{N}}{\nabla} g^{n}$, then $H\left(\left[\left(x_{n}\right)\right], 0\right)=$ $[(0,0, \ldots)]$, and $H\left(\left[\left(x_{n}\right)\right], 1\right)=[(1,1, \ldots)]$ for every $\left[\left(x_{n}\right)\right] \in \tilde{I}$. Then for every $\left[\left(x_{n}\right)\right]$, a map $I \rightarrow \tilde{I}$ defined by $t \mapsto H\left(\left[\left(x_{n}\right)\right], t\right)$ is a path from $[(0,0, \ldots)]$ to $[(1,1, \ldots)]$ in $\tilde{I}$, and that is a contradiction because $\tilde{I}$ is totally disconnected.

Now, let $\left[\left(\left[f^{n}\right]\right)\right] \in$ pro* $^{*}-H T o p((X),(Y))$. Recall, $\left(\left[f^{n}\right]\right)$ and $\left(\left[f^{\prime n}\right]\right)$ are equivalent if there is an $n_{0} \in \mathbb{N}$ such that $\left[f^{n}\right]=\left[f^{\prime n}\right]$ for every $n \geqslant n_{0}$.

We claim that $\left(\left[f^{n}\right]\right)$ and $\left(\left[f^{\prime n}\right]\right)$ induce the same box-homotopy class $\left[\nabla_{\mathbb{N}} f^{n}\right]_{\square}$. Indeed, let $\left(f^{n}\right)$ and $\left(f^{\prime n}\right)$ be two sequences of continuous maps between spaces $X$ and $Y$ such that $f^{n} \sim f^{\prime n}$ by a homotopy $H_{n}$ for every $n \geqslant n_{0}$. We can define a mapping $H: \square_{\mathbb{N}}(X \times I) \rightarrow \underset{\mathbb{N}}{\square}$ by

$$
q_{n} \circ H= \begin{cases}H_{n}, & n \geqslant n_{0}, \\ H_{n_{0}}, & n \leqslant n_{0},\end{cases}
$$

where $q_{n}: \square_{\mathbb{N}} Y \rightarrow Y$ is a projection. $H$ is continuous, and $H \circ i$, where $i$ is the mapping from Lemma 5.2, induces a box homotopy $\tilde{H}: \tilde{X} \times \underset{\mathbb{N}}{\square} I \rightarrow \tilde{Y}, \tilde{H}\left(\left[\left(x_{n}\right)\right],\left(t_{n}\right)\right)=$ $\left[H\left(\left(x_{n}, t_{n}\right)\right)\right]$, between $\underset{\mathbb{N}}{\nabla} f^{n}$ and $\underset{\mathbb{N}}{\nabla} f^{\prime n}$. Indeed,

$$
\begin{aligned}
& \tilde{H}\left(\left[\left(x_{n}\right)\right],(0,0, \ldots)\right)=\left[\left(f^{n}\left(x_{n}\right)\right)\right], \\
& \tilde{H}\left(\left[\left(x_{n}\right)\right],(1,1, \ldots)\right)=\left[\left(f^{\prime n}\left(x_{n}\right)\right)\right] .
\end{aligned}
$$

Therefore, by the correspondence pro $^{*}-H T o p \rightarrow$ pro- $H_{\square}$ Top defined by

$$
\tilde{R}\left(\left(X_{\lambda},\left[p_{\lambda \lambda^{\prime}}\right], \Lambda\right)\right)=\left(\tilde{X}_{\lambda},\left[\underset{\mathbb{N}}{\nabla} p_{\lambda \lambda^{\prime}}\right]_{\square}, \Lambda\right)
$$

for $\left(X_{\lambda},\left[p_{\lambda \lambda^{\prime}}\right], \Lambda\right) \in O b\left(\right.$ pro $\left.^{*}-H T o p\right)$, and

$$
\tilde{R}\left(\left[\left(f,\left[f_{\mu}^{n}\right]\right)\right]\right)=\left[\left(f,\left[\underset{\mathbb{N}}{\nabla} f_{\mu}^{n}\right]_{\square}\right)\right]
$$

for $\left[\left(f,\left[f_{\mu}^{n}\right]\right)\right] \in \operatorname{Mor}\left(\right.$ pro $\left.^{*}-H T o p\right)$, the function $\tilde{R}$ is well defined. By chasing an appropriate diagram one can easily check that $\left[\left(f,\left[{ }_{\mathbb{N}}^{\nabla} f^{n}\right]_{\square}\right)\right]$ is indeed a morphism of pro- $H_{\square} T o p$ and that functorial properties are valid.

The functor $\tilde{R}$ is neither faithful nor full. For instance, morphisms $\boldsymbol{f}=\left[\left(f^{n}\right)\right], \boldsymbol{g}=$ $\left[\left(g^{n}\right)\right] \in$ pro $^{*}-H T o p((X),(Y))$ such that $f^{n}$ and $g^{n}$ are box-homotopic but not homotopic for every $n \in \mathbb{N}$, are different, and, by $5.6, \tilde{R}(\boldsymbol{f})=\tilde{R}(\boldsymbol{g})$. Also, morphisms in pro- $H_{\square}$ Top between inverse systems of non- $P$-spaces do not come from pro $^{*}-H T o p$ under $\tilde{R}$.

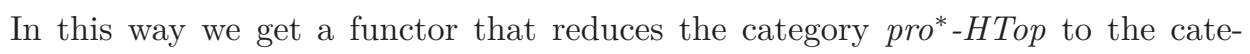
gory pro- $H_{\square}$ Top, making the objects more complex, but the number of morphisms is significantly reduced, which makes analyzing and constructing isomorphisms easier.

We can go further on and in a similar manner define a certain correspondence $\tilde{R}: p^{*}{ }^{*} H_{\square} T o p \rightarrow$ pro- $H_{\square}$ Top by

$$
\tilde{R}\left(\left(X_{\lambda},\left[p_{\lambda \lambda^{\prime}}\right]_{\square}, \Lambda\right)\right)=\left(\tilde{X}_{\lambda},\left[\nabla_{\mathbb{N}} p_{\lambda \lambda^{\prime}}\right]_{\square}, \Lambda\right)
$$


for $\left(X_{\lambda},\left[p_{\lambda \lambda^{\prime}}\right]_{\square}, \Lambda\right) \in O b\left(\right.$ pro $\left.^{*}-H_{\square} T o p\right)$, and

$$
\tilde{R}\left(\left[\left(f,\left[f_{\mu}^{n}\right]_{\square}\right)\right]\right)=\left[\left(f,\left[\nabla_{\mathbb{N}} f_{\mu}^{n}\right]_{\square}\right)\right]
$$

for $\left[\left(f,\left[f_{\mu}^{n}\right]_{\square}\right)\right] \in \operatorname{Mor}\left(\right.$ pro $\left.^{*}-H_{\square} T o p\right)$. It proves out that $\tilde{R}$ is also a well defined functor.

Now, if we apply the known coarse shape theory techniques, recalling that every topological space admits a polyhedral resolution, the sequence of functors

$$
\begin{aligned}
& \text { HTop } \stackrel{\underline{S}}{\longrightarrow} \text { pro-HPol } \stackrel{\underline{J}}{\longrightarrow} \text { pro* }-H P o l \stackrel{\tilde{R}}{\longrightarrow} \text { pro }-H_{\square} \text { Top } \stackrel{\underline{J}}{\longrightarrow} \\
& \text { pro* }-H_{\square} \text { Top } \stackrel{\tilde{R}}{\longrightarrow} \text { pro- } H_{\square} \text { Top } \stackrel{\underline{J}}{\longrightarrow} \\
& \text { pro* }-H_{\square} \text { Top } \stackrel{\tilde{\tilde{R}}}{\longrightarrow} \text { pro }-H_{\square} \text { Top } \stackrel{\underline{J}}{\longrightarrow} \ldots
\end{aligned}
$$

induces a sequence of classifications coarser than the coarse shape type. Here, $\underline{S}$ acts as a shape functor by fixing an expansion of a topological space.

\section{References}

[1] P. Bankston, Ultraproducts in topology, Gen. Topol. Appl. 7 (1977) 283-308.

[2] N. Koceić Bilan, N. Uglešić, The coarse shape, Glas. Mat. 42 (62) (2007) 145187.

[3] K. Kunen Paracompactness of box products of compact spaces, Trans. Amer. Math. Soc. 240 (1978) 307-316.

[4] S. Mardešić, J. Segal, Shape Theory, North-Holland, Amsterdam, 1982.

[5] A.K. Misra A topological view of P-spaces, Gen. Topol. Appl. 2 (1972) 349-362.

[6] M.E. Rudin, Lectures on Set Theoretic Topology, American Mathematical Society, 1975 .

Nikola Koceić Bilan koceic@pmfst.hr

Faculty of Science, University of Split, Ruđera Boškovića 33, 21000 Split, Croatia

Ivančica Mirošević ivancica.mirosevic@fesb.hr

Faculty of Electrical Engineering, Mechanical Engineering and Naval Architecture, University of Split, Ruđera Boškovića 32, 21000 Split, Croatia 